\title{
Triple monogenic functions and higher spin Dirac operators
}

\author{
F. Brackx*, D. Eelbode ${ }^{\ddagger}$, T. Raeymaekers*, L. Van de Voorde* \\ * Clifford Research Group, Department of Mathematical Analysis, \\ Faculty of Engineering, Ghent University, Belgium \\ ¥ Department of Mathematics and Computer Science, University of Antwerp, \\ Campus Middelheim, G-Building, Middelheimlaan 1, 2020 Antwerpen, Belgium
}

\begin{abstract}
In the Clifford analysis context a specific type of solutions for the higher spin Dirac operators $\mathcal{Q}_{k, l}(k \geq l \in \mathbb{N})$ is studied; these higher spin Dirac operators can be seen as generalizations of the classical Rarita-Schwinger operator. To that end subspaces of the space of triple monogenic polynomials are introduced and their algebraic structure is investigated. Also a dimensional analysis is carried out.
\end{abstract}

\section{Introduction}

Recently it has become clear that Clifford analysis offers an elegant framework to study higher spin Dirac operators from a function theoretical point of view. These operators can be defined as $\operatorname{Spin}(m)$-invariant elliptic differential operators acting between spaces of functions of several vector variables, taking their values in a polynomial model for an irreducible $\operatorname{Spin}(m)$-module. They may be seen as far-reaching generalizations of both the Dirac operator and the Rarita-Schwinger operator. Both these operators were originally inspired by physics, but in Clifford analysis one studies them from a completely different point of view. Note that each one of these operators, whose existence is encoded in Fegan's celebrated paper [12], can be constructed using the method of generalized gradients, see e.g. $[5,22,1]$. The Dirac operator has been, until now, the most studied operator in Clifford analysis: we refer to e.g. [10] for a historical overview and a short introduction and to e.g. $[2,9,14,15]$ for a detailed account of this research field. For the case of the RaritaSchwinger operators we refer to [6,7]; in these papers techniques from representation theory and from classical Clifford analysis were successfully applied to construct these operators explicitly and to describe their polynomial null solutions. In a series of recent papers, see e.g. $[11,3,4]$, further generalizations of these higher spin Dirac operators (HSD operators) were studied in an attempt to describe properties of HSD operators within an encompassing framework. Doing so, we have been able to isolate a few related problems which need to be solved first in order to formulate an adequate theory. This paper deals with one of these problems: apparently spaces of triple monogenic functions, and certain subspaces thereof, have to be well-understood in order to describe the space of polynomial solutions for HSD operators. We will introduce specific (sub)spaces of triple monogenic functions and study them from an algebraic point of view. This will enable us to obtain an explicit description of a specific type of polynomial null solutions for the HSD-operators $\mathcal{Q}_{k, l}$, introduced in [3], and defined in Section 2. 


\section{Higher spin operators and their null solutions}

Consider the Clifford algebra $\mathbb{R}_{m}$ generated by an orthonormal basis $\left(\underline{e}_{1}, \ldots, \underline{e}_{m}\right)$ for the vector space $\mathbb{R}^{m}$, and its complexified version $\mathbb{C}_{m}=\mathbb{R}_{m} \otimes \mathbb{C}$. The scalars and the vectors are thought of as being embedded in a natural way in the Clifford algebra. The multiplication in $\mathbb{R}_{m}$ and $\mathbb{C}_{m}$ is governed by the relations $\underline{e}_{i} \underline{e}_{j}+\underline{e}_{j} \underline{e}_{i}=-2 \delta_{i j}, i, j=1, \ldots, m$. Let $\mathbb{S}$ (respectively $\mathbb{S}^{ \pm}$) be the unique spinor space (respectively the space of positive and negative spinors), which can be realized as a primitive ideal inside the complex Clifford algebra $\mathbb{C}_{m}$ in case $m$ is odd (respectively $m$ is even). Unless otherwise stated, we will often disregard the parity of the spinors in even dimension and so in both cases speak about the space of spinors.

The classical Dirac operator $\underline{\partial}_{x}=\sum_{j=1}^{m} \underline{e}_{j} \partial_{x_{j}}$ on $\mathbb{R}^{m}$ is an elliptic differential operator acting between spaces of smooth spinor-valued functions. It is invariant w.r.t. to the regular representation $L(s)$ of $\operatorname{Spin}(m)$ on $\mathcal{C}^{\infty}\left(\mathbb{R}^{m} ; \mathbb{S}\right)$ given by $L(s)[f(\underline{x})]:=s f(\bar{s} \underline{x} s), s \in \mathbb{S}$, where the bar denotes the Clifford conjugation. Moreover it factorizes the Laplace operator $\Delta_{x}$ on $\mathbb{R}^{m}$ in the sense that $\underline{\partial}_{x}^{2}=-\Delta_{x}$. Null solutions of the Dirac operator are called monogenic functions, and the vector space $\mathcal{M}_{k}\left(\mathbb{R}^{m} ; \mathbb{S}\right)$ of $k$-homogeneous monogenic polynomials defines a polynomial model for the irreducible $\operatorname{Spin}(m)$-module with highest weight

$$
(k)^{\prime}:=\left(k+\frac{1}{2}, \frac{1}{2}, \cdots, \pm \frac{1}{2}\right) .
$$

Note that the minus sign only applies to the case of even dimension, and will be omitted unless explicitly needed. Also mind the notation: from now one we will use an accent to denote a highest weight which can be seen as the Cartan product of an integer weight with the highest weight for the spinor space. More generally, it is known that arbitrary irreducible (half-integer) $\operatorname{Spin}(m)$-modules can be described as spinor-valued tensors satisfying certain symmetry conditions expressed in terms of Young diagrams, see e.g. [13, 17], but they can also be represented as vector spaces of polynomials. In e.g. $[8,14]$ it was shown that such modules of half-integer weight can be realized in terms of monogenic polynomials of several vector variables. In what follows, $N \in \mathbb{N}$ and $\langle\cdot, \cdot\rangle$ denotes the Euclidean inner product.

Definition 1 An $\mathbb{S}$-valued function $f: \mathbb{R}^{N m} \rightarrow \mathbb{S}:\left(\underline{u}_{1}, \ldots, \underline{u}_{N}\right) \mapsto f\left(\underline{u}_{1}, \ldots, \underline{u}_{N}\right)$ is simplicial monogenic if the following conditions are satisfied:

$$
\underline{\partial}_{\underline{u}_{i}} f=0 \quad(i=1, \ldots, N) \quad \text { and } \quad\left\langle\underline{u}_{i}, \underline{\partial}_{\underline{u}_{j}}\right\rangle f=0 \quad(1 \leq i<j \leq N) .
$$

The vector space of $\mathbb{S}$-valued simplicial monogenic polynomials which, for each $i=1, \ldots, N$, are $\lambda_{i}$-homogeneous in the variable $\underline{u}_{i}$, will be denoted $\mathcal{S}_{\lambda_{1}, \ldots, \lambda_{N}}$ (with $\lambda_{1} \geq \ldots \geq \lambda_{N}$ from now on, i.e. satisfying the dominant weight condition).

It was shown in [8] that the space $\mathcal{S}_{\lambda_{1}, \ldots, \lambda_{N}}$ is characterized by the highest weight $\left(\lambda_{1}, \ldots, \lambda_{N}\right)^{\prime}$ with respect to the regular representation on polynomials in several vector variables. Note that the case where the last entry of the highest weight is a non-trivial number (i.e. $2 N=n$ ), is always somewhat special: one then needs an extra condition to characterize the modules. This is related to the so-called massless field operator and their generalizations, see e.g. [18].

Taking $N=2$, for every $k \geq l \in \mathbb{N}$ the HSD operator $\mathcal{Q}_{k, l}$ is uniquely defined, up to a multiplicative constant, as the invariant differential operator acting as

$$
\mathcal{Q}_{k, l}: \mathcal{C}^{\infty}\left(\mathbb{R}^{m} ; \mathcal{S}_{k, l}\right) \rightarrow \mathcal{C}^{\infty}\left(\mathbb{R}^{m} ; \mathcal{S}_{k, l}\right): f(\underline{x} ; \underline{u}, \underline{v}) \mapsto \mathcal{Q}_{k, l} f(\underline{x} ; \underline{u}, \underline{v}) .
$$


An explicit expression for this operator was found in [3], using techniques from representation theory.

Definition 2 For every $k \geq l$, the operator $\mathcal{Q}_{k, l}$ is defined by

$$
\mathcal{Q}_{k, l} f=\left(\mathbf{1}+\frac{\underline{u} \underline{\partial}_{u}}{m+2 k-2}+\frac{\underline{v} \underline{\partial}_{v}}{m+2 l-4}-2 \frac{\underline{u}\left\langle\underline{v}, \underline{\partial}_{u}\right\rangle \underline{\partial}_{v}}{(m+2 k-2)(m+2 l-4)}\right) \underline{\partial}_{x} f .
$$

Note that in case $l=0, \mathcal{Q}_{k, 0}$ reduces to the Rarita-Schwinger operator $\mathcal{R}_{k}$, see [6]:

$$
\mathcal{Q}_{k, 0} f=\mathcal{R}_{k} f=\left(\mathbf{1}+\frac{\underline{u} \underline{\partial}_{u}}{m+2 k-2}\right) \underline{\partial}_{x} f
$$

Closely related to the operators $\mathcal{Q}_{k, l}$ are the so-called twistor operators

$$
\mathcal{T}_{k, l-1}^{k, l}: \mathcal{C}^{\infty}\left(\mathbb{R}^{m}, \mathcal{S}_{k, l}\right) \rightarrow \mathcal{C}^{\infty}\left(\mathbb{R}^{m}, \mathcal{S}_{k, l-1}\right)
$$

and (in case $k>l$ )

$$
\mathcal{T}_{k-1, l}^{k, l}: \mathcal{C}^{\infty}\left(\mathbb{R}^{m}, \mathcal{S}_{k, l}\right) \rightarrow \mathcal{C}^{\infty}\left(\mathbb{R}^{m}, \mathcal{S}_{k-1, l}\right) .
$$

These $\operatorname{Spin}(m)$-invariant operators, whose existence is also described by a result by Fegan, are no longer elliptic, but they play a crucial role in obtaining null solutions of the HSD operators by means of an inductive argument. In the case of the classical Rarita-Schwinger operator $\mathcal{R}_{k}$ it was shown in e.g. [6], that it has two types of (homogeneous) polynomial null solutions: a first type consisting of double monogenic functions, and a second type consisting of solutions which can be seen as images under the inverse of the twistor operator of null solutions of the operator $\mathcal{R}_{k-1}$. The HSD operator $\mathcal{Q}_{k, l}$ shows a similar behaviour. First, there are null solutions of the operator $\mathcal{Q}_{k, l}$ which can be obtained through the inverse of the twistor operators mentioned above. However note that this is not a straightforward generalization of the case $l=0$; one needs a careful analysis to know precisely which summands in the kernels of the operators $\mathcal{Q}_{k, l-1}$ and $\mathcal{Q}_{k-1, l}$ can actually be inverted. We refer to [4] for more details on this first type of solutions. A second type of null solutions of the operator $\mathcal{Q}_{k, l}$ is obtained in terms of so-called triple monogenic polynomials.

Definition 3 For each 3-tuple $(p, q, r)$ of positive integers, the space $\mathcal{M}_{p, q, r}\left(\mathbb{R}^{3 m} ; \mathbb{S}\right)$ of socalled triple monogenics, consists of $\mathbb{S}$-valued polynomials in three vector variables $(\underline{x}, \underline{u}, \underline{v})$, homogeneous of degree $(p, q, r)$ in $(\underline{x}, \underline{u}, \underline{v})$ respectively, satisfying $\underline{\partial}_{x} P=\underline{\partial}_{u} P=\underline{\partial}_{v} P=0$.

In this paper, we will also need a related function space, which is obtained by intersecting $\mathcal{M}_{p, q, r}\left(\mathbb{R}^{3 m} ; \mathbb{S}\right)$ with the kernel of the skew Euler operator $\left\langle\underline{u}, \underline{\partial}_{v}\right\rangle$. This extra condition is needed to ensure that we are dealing with polynomials which, for each $\underline{x}$ take their values in $\mathcal{S}_{k, l}$. Hence the following definition.

Definition 4 For each 3-tuple $(p, q, r)$ of positive integers, with $p \geq q \geq r$, we define the space $\mathcal{M}_{p, q, r}^{s}\left(\mathbb{R}^{3 m} ; \mathbb{S}\right)$ of so-called skew triple monogenic polynomials, by

$$
\mathcal{M}_{p, q, r}^{s}\left(\mathbb{R}^{3 m} ; \mathbb{S}\right):=\left\{P(\underline{x} ; \underline{u}, \underline{v}) \in \mathcal{M}_{p, q, r}\left(\mathbb{R}^{3 m} ; \mathbb{S}\right):\left\langle\underline{u}, \underline{\partial}_{v}\right\rangle P=0\right\} .
$$

In the next section, we will study these spaces from an algebraic point of view. In the meantime, we can already mention the following crucial fact: for any $h \geq k \geq l$, we have that $\mathcal{M}_{h, k, l}^{s} \subset \operatorname{Ker}_{h} \mathcal{Q}_{k, l}$, where from now on we reserve the notation $\operatorname{Ker}_{h} \mathcal{D}$ for the space of $h$-homogeneous polynomial null solutions of the operator $\mathcal{D}$. This is the second type of solutions of the operator $\mathcal{Q}_{k, l}$ and the aim of this paper is to determine the structure of this space and to calculate its dimension. 


\section{Skew triple monogenic polynomials}

We consider the classical Lie algebra $\mathfrak{g l}_{3}$, spanned by the basis elements $E_{i j}, 1 \leq i, j \leq 3$, satisfying the following commutator relations:

$$
\left[E_{i j}, E_{k l}\right]=\delta_{k j} E_{i l}-\delta_{i l} E_{k j} .
$$

As is well-known, finite-dimensional irreducible representations for this Lie algebra are in a one-to-one correspondence with 3 -tuples $\left(\lambda_{1}, \lambda_{2}, \lambda_{3}\right)$ of non-negative integers numbers satisfying $\lambda_{1} \geq \lambda_{2} \geq \lambda_{3} \geq 0$. This 3 -tuple is called the highest weight $\lambda$ of the representation, and we will denote by $\mathbb{V}(\lambda)$ the irreducible representation corresponding to $\lambda$. This module contains a unique (up to a multiplicative constant) highest weight vector $\underline{v}_{\lambda}$ such that $E_{i i} \underline{v}_{\lambda}=\lambda_{i} \underline{v}_{\lambda}$ and $E_{i j} \underline{v}_{\lambda}=0$ for $i<j$. As we will see further on, there is a nice polynomial model for these modules within the setting of Clifford analysis. This is possible because there is a model for $\mathfrak{g l}_{3}$ in terms of specific differential operators in several vector variables. It can indeed be verified that the following isomorphism, compactly denoted in matrix notation by

$$
\left(\begin{array}{ccc}
E_{11} & E_{12} & E_{13} \\
E_{21} & E_{22} & E_{23} \\
E_{31} & E_{32} & E_{33}
\end{array}\right) \leftrightarrow\left(\begin{array}{ccc}
\mathbb{E}_{x}+\frac{m}{2} & \left\langle\underline{x}, \underline{\partial}_{u}\right\rangle & \left\langle\underline{x}, \underline{\partial}_{v}\right\rangle \\
\left\langle\underline{u}, \underline{\partial}_{x}\right\rangle & \mathbb{E}_{u}+\frac{m}{2} & \left\langle\underline{u}, \underline{\partial}_{v}\right\rangle \\
\left\langle\underline{v}, \underline{\partial}_{x}\right\rangle & \left\langle\underline{v}, \underline{\partial}_{u}\right\rangle & \mathbb{E}_{v}+\frac{m}{2}
\end{array}\right),
$$

where $\mathbb{E}_{y}$ denotes the Euler operator with respect to the variable $y=\underline{x}, \underline{u}$ or $\underline{v}$, gives an alternative realization for the algebra $\mathfrak{g l}_{3}$. From now on, we will sometimes use $E_{i j}$ to denote the operator corresponding to this element under the isomorphism above.

Recall that a triple monogenic polynomial $P$ is called simplicial monogenic if moreover the conditions $E_{i j} P=0$, for all $i<j$, are satisfied. The following lemma can then be proved:

Lemma 1 Each element $E_{i j}$ of the Lie algebra $\mathfrak{g l}_{3}$ acts as an endomorphism on the space of triple monogenic polynomials.

The fact that the operators defining simplicial monogenic polynomials are the positive root vectors in $\mathfrak{g l}_{3}$, suggests considering the whole vector space $\mathcal{S}_{\lambda_{1}, \lambda_{2}, \lambda_{3}}$ as a highest weight vector for the Lie algebra $\mathfrak{g l}_{3}$ (where we assume that, after a possible reordering of the vector variables, $\lambda_{1} \geq \lambda_{2} \geq \lambda_{3}$ ). In view of Lemma 1 , we thus have:

Proposition 1 For each 3-tuple of positive integers $\left(\lambda_{1}, \lambda_{2}, \lambda_{3}\right)$ satisfying $\lambda_{1} \geq \lambda_{2} \geq \lambda_{3}$, the vector space $\mathcal{S}_{\lambda_{1}, \lambda_{2}, \lambda_{3}}$ of simplicial monogenics in three vector variables, generates a finite-dimensional irreducible module for the Lie algebra $\mathfrak{g l}_{3}$.

We will label this module by

$$
\mathbb{V}\left(\mathcal{S}_{\lambda_{1}, \lambda_{2}, \lambda_{3}}\right)=\mathbb{V}\left(\lambda_{1}+\frac{m}{2}, \lambda_{2}+\frac{m}{2}, \lambda_{3}+\frac{m}{2}\right) .
$$

It is clear that for any 3 -tuple of positive integers $\left(\lambda_{1}, \lambda_{2}, \lambda_{3}\right)$ satisfying $\lambda_{1} \geq \lambda_{2} \geq \lambda_{3}$, we have that $\mathbb{V}\left(\mathcal{S}_{\lambda_{1}, \lambda_{2}, \lambda_{3}}\right) \subset \mathcal{M}\left(\mathbb{R}^{3 m}, \mathbb{S}\right)$, the latter denoting the space of all triple monogenic polynomials. This space is an invariant (and highly reducible) module under the spin group $\operatorname{Spin}(m)$ and a natural problem consists in decomposing this module into irreducible modules for the spin group (i.e. basic building blocks). 
Let us first illustrate this for the case of two vector variables: in $[6,7]$ it was shown that the space $\mathcal{M}_{k, l}\left(\mathbb{R}^{2 m} ; \mathbb{S}\right)$ of $(k, l)$-homogeneous double monogenic polynomials in two vector variables $(\underline{x}, \underline{u}) \in \mathbb{R}^{2 m}$ decomposes as

$$
\mathcal{M}_{k, l}\left(\mathbb{R}^{2 m} ; \mathbb{S}\right)=\bigoplus_{j=0}^{l}\left\langle\underline{u}, \underline{\partial}_{x}\right\rangle^{j} \mathcal{S}_{k+j, l-j},
$$

which is precisely the type of result we are looking for. As the spaces of simplicial monogenics define a model for irreducible $\operatorname{Spin}(m)$-modules with half-integer highest weight, see e.g. $[8,14]$, it will therefore be sufficient to decompose the space $\mathcal{M}_{p, q, r}\left(\mathbb{R}^{3 m} ; \mathbb{S}\right)$ into spaces of simplicial monogenics. To that end we need to find all $\operatorname{Spin}(m)$-invariant mappings from spaces of simplicial monogenics into the space of triple monogenics. From the theory on Howe dual pairs we know that the only candidates for this are combinations of the basic invariants underlying Clifford analysis in three vector variables. These basic invariants are the operators generating the Lie superalgebra $\mathfrak{o s p}(1 \mid 6)=\mathfrak{g}_{0} \oplus \mathfrak{g}_{1}$, with even subalgebra $\mathfrak{g}_{0}=\mathfrak{s p}(6)$ spanned by the 21 elements

$$
\begin{aligned}
& \left\{E_{i j}: 1 \leq i, j \leq 3\right\} \oplus\left\{|\underline{x}|^{2},|\underline{u}|^{2},|\underline{v}|^{2}, \Delta_{x}, \Delta_{u}, \Delta_{v}\right\} \\
& \oplus\left\{\langle\underline{x}, \underline{u}\rangle,\langle\underline{x}, \underline{v}\rangle,\langle\underline{u}, \underline{v}\rangle,\left\langle\underline{\partial}_{x}, \underline{\partial}_{u}\right\rangle,\left\langle\underline{\partial}_{x}, \underline{\partial}_{v}\right\rangle,\left\langle\underline{\partial}_{u}, \underline{\partial}_{v}\right\rangle\right\}
\end{aligned}
$$

and the odd subspace

$$
\mathfrak{g}_{1}=\left\{\underline{x}, \underline{u}, \underline{v}, \underline{\partial}_{x}, \underline{\partial}_{u}, \underline{\partial}_{v}\right\} .
$$

A crucial result in the general theory of Lie superalgebras is the PBW-theorem, which states that any combination of these invariants, i.e. any element in the universal enveloping algebra $U(\mathfrak{o s p}(1 \mid 6))$, can always be reordered (using the algebraic relations) so that we obtain (a sum of) elements of the form

$$
X_{1}^{a_{1}} X_{2}^{a_{2}} \cdots X_{26}^{a_{26}} X_{27}^{a_{27}} \in U(\mathfrak{o s p}(1 \mid 6)),
$$

with $X_{j}$ the generators for $\mathfrak{o s p}(1 \mid 6)$ and $a_{j} \in \mathbb{Z}_{+}$. If we now choose an ordering on the generators in such a way that

(i) we first list all combinations involving only the vector variables (type A)

(ii) we then list all the elements in $\mathfrak{g l}_{3}$ (type B)

(iii) we finally list the combinations involving only the Dirac operators (type C),

it becomes clear that the only elements in $U(\mathfrak{o s p}(1 \mid 6))$ which can be used, are elements in $U\left(\mathfrak{g l}_{3}\right)$. Indeed, combinations involving type $\mathrm{C}$ operators will act trivially on the space of simplicial monogenics, whereas combinations involving type A operators will always belong to the Fischer complement of the space of triple monogenics. This latter statement is based on the fact that

$$
\mathcal{P}_{p, q, r}=\mathcal{M}_{p, q, r} \oplus\left(\underline{x} \mathcal{P}_{p-1, q, r}+\underline{u} \mathcal{P}_{p, q-1, r}+\underline{v} \mathcal{P}_{p, q, r-1}\right),
$$

the sum between brackets obviously not being a direct sum. We are then led to the following important conclusion, which will be dimensionally verified in the next section. 
Proposition 2 In order to decompose the space $\mathcal{M}_{p, q, r}\left(\mathbb{R}^{3 m} ; \mathbb{S}\right)$ into irreducible modules for the spin group Spin $(m)$, it suffices to select all the weight spaces having the correct degree of homogeneity inside each of the $\mathfrak{g l}_{3}$-modules $\mathbb{V}\left(\mathcal{S}_{\lambda_{1}, \lambda_{2}, \lambda_{3}}\right)$ generated by the spaces of simplicial monogenics.

Next, we are interested in the spaces of skew triple monogenic polynomials; recall that for each 3-tuple $(p, q, r)$ of positive integers with $p \geq q \geq r$, the space of skew triple monogenics is given by

$$
\mathcal{M}_{p, q, r}^{s}:=\mathcal{M}_{p, q, r} \cap \operatorname{Ker}\left\langle\underline{u}, \underline{\partial}_{v}\right\rangle .
$$

In order to describe this space algebraically, we will use branching rules from $\mathfrak{g l}_{3}$ to $\mathfrak{g l}_{2}$, see e.g. [20]. Let us first define the subspace $\mathbb{V}(\lambda)^{+}$containing $\mathfrak{g l}_{2}$-highest weight vectors in $\mathbb{V}(\lambda)$ :

$$
\mathbb{V}(\lambda)^{+}=\left\{\eta \in \mathbb{V}(\lambda): E_{23} \eta=0\right\} .
$$

Given a weight $\mu\left(\mu_{2}, \mu_{3}\right)$ for the smaller algebra $\mathfrak{g l}_{2}$, we then introduce the set containing the weight spaces in $\mathbb{V}(\lambda)$ realizing a copy of the module for the algebra $\mathfrak{g l}_{2}$ with highest weight $\mu$ :

$$
\mathbb{V}(\lambda)_{\mu}^{+}=\left\{\eta \in \mathbb{V}(\lambda)^{+}: E_{i i} \eta=\mu_{i} \eta, i=2,3\right\} .
$$

A classical result then tells us that $\mathbb{V}(\lambda)_{\mu}^{+}$is either trivial or one-dimensional, and that

$$
\operatorname{dim}\left(\mathbb{V}(\lambda)_{\mu}^{+}\right)=1 \Leftrightarrow\left(\lambda_{a-1}-\mu_{a}, \mu_{a}-\lambda_{a}\right) \in \mathbb{Z}_{+}^{2},
$$

for $a=2,3$. In its turn this tells us, given a space $\mathbb{V}\left(\mathcal{S}_{\lambda_{1}, \lambda_{2}, \lambda_{3}}\right)$, how many of the weight spaces can actually be selected. Indeed, applying this result shows that

$$
\left.\mathbb{V}\left(\mathcal{S}_{\lambda_{1}, \lambda_{2}, \lambda_{3}}\right)\right|_{\mathfrak{g l}_{2}} ^{\mathfrak{g l _ { 3 }}}=\bigoplus_{\mu} \mathbb{V}\left(\mathcal{S}_{\mu_{2}, \mu_{3}}\right)=\bigoplus_{i, j} \mathbb{V}\left(\mathcal{S}_{\lambda_{2}+i, \lambda_{3}+j}\right),
$$

where $0 \leq i \leq \lambda_{1}-\lambda_{2}$ and $0 \leq j \leq \lambda_{2}-\lambda_{3}$, and in each of these $\mathfrak{g l}_{2}$-modules we then only need to pick up the highest weight vector. This element, which is of course a polynomial in $\mathbb{V}\left(\mathcal{S}_{\lambda_{1}, \lambda_{2}, \lambda_{3}}\right)$, will belong to the space $\mathcal{M}_{\lambda_{1}, \lambda_{2}, \lambda_{3}}^{s}$.

Finally, we would like to describe how these highest weight vectors for the $\mathfrak{g l}_{2}$-modules are embedded into the space $\mathbb{V}\left(\mathcal{S}_{\lambda_{1}, \lambda_{2}, \lambda_{3}}\right)$. This will enable us to explicitly decompose the space of skew triple monogenics. This can be done using the so-called raising and lowering operators, for which we again refer to e.g. [20].

\section{Dimensional analysis}

\subsection{Dimension of $\mathcal{M}_{p, q, r}$}

Our aim is to find a decomposition of the space of triple monogenics $\mathcal{M}_{p, q, r}$, similar to the decomposition (2) for $\mathcal{M}_{k, l}$. This requires information on the weights in a representation of the Lie algebra $\mathfrak{s l}_{3}$ for which it may be proved that

$$
\mathfrak{s l}_{3} \cong \operatorname{span}\left\{\left\langle\underline{x}, \underline{\partial}_{u}\right\rangle,\left\langle\underline{u}, \underline{\partial}_{x}\right\rangle,\left\langle\underline{x}, \underline{\partial}_{v}\right\rangle,\left\langle\underline{v}, \underline{\partial}_{x}\right\rangle,\left\langle\underline{u}, \underline{\partial}_{v}\right\rangle,\left\langle\underline{v}, \underline{\partial}_{u}\right\rangle, \mathbb{E}_{x}-\mathbb{E}_{u}, \mathbb{E}_{x}-\mathbb{E}_{v}\right\} .
$$

First, we summarize some basic results of representations of $\mathfrak{s l}_{3}$; for details we refer to e.g. $[13,16]$. Let $L_{1}, L_{2}, L_{3}$ be linear functionals on $\mathbb{C}$ such that $L_{1}+L_{2}+L_{3}=0$. In order to 
visualize the action of $\mathfrak{s l}_{3}$, it is convenient to identify these linear functionals with elements of $\mathbb{C}$. This determines a triangular grid.

The Weyl group is a symmetry group of the weights of any (finite-dimensional) representation. In case of $\mathfrak{s l}_{3}$, it consists of six elements: the identity, clockwise and counterclockwise rotations by $120^{\circ}$ and three reflections. If $\mu$ is a weight for a representation, then the action of the Weyl group on $\mu$ is also a weight for this representation with the same multiplicity. Any weight $\lambda$ of a representation of $\mathfrak{s l}_{3}$ can be denoted by $\lambda=a L_{1}-b L_{3}=:[a, b]$ with $a, b \in \mathbb{Z}$. The set $a L_{1}-b L_{3}$ with $a \geq 0$ and $b \geq 0$ is called the (closed) Weyl chamber (relative to $L_{1}, L_{2}, L_{3}$ ). Every highest weight lies in the Weyl chamber.

The last two operators in (4) are elements of the Cartan subalgebra of $\mathfrak{s l}_{3}$. The action of the other operators on a weight $\lambda$, is visualized in Figure 1. Note that $\left[\left\langle\underline{u}, \underline{\partial}_{u}\right\rangle,\left\langle\underline{u}, \underline{\partial}_{x}\right\rangle\right]=$ $\left\langle\underline{v}, \underline{\partial}_{x}\right\rangle,\left[\left\langle\underline{u}, \underline{\partial}_{x}\right\rangle,\left\langle\underline{v}, \underline{\partial}_{x}\right\rangle\right]=0$ and $\left[\left\langle\underline{v}, \underline{\partial}_{u}\right\rangle,\left\langle\underline{v}, \underline{\partial}_{x}\right\rangle\right]=0$.


Figure 1: Action of $\mathfrak{s l}_{3}$ on the weight $\lambda$

As opposed to the case of $\mathfrak{s l}_{2}$, not every weight in a representation of $\mathfrak{s l}_{3}$ appears with multiplicity 1 . It can be shown that in a representation of $\mathfrak{s l}_{3}$ the multiplicities of the weights obey the following simple pattern: they occur in "rings" where the rings towards the outside are hexagons and the rings towards the inside are triangles. The weights in the outermost ring have multiplicity 1 . The multiplicities then increase by 1 each time one moves one ring inwards, until the rings become triangles, at which point the multiplicities stabilise. As an example, Figure 2 shows one hexagon of weights of multiplicity 1 and a triangle of weights of multiplicity 2 .

It has been mentioned before that the space $\mathcal{S}_{p, q, r}$ of simplicial monogenics is an irreducible $\operatorname{Spin}(m)$-module with highest weight $(p, q, r)^{\prime}$. As a corollary of Proposition 1, $\mathcal{S}_{p, q, r}$ is a highest weight vector for $\mathfrak{s l}_{3}$. It can be shown, see e.g. [21], that this highest weight vector corresponds to a unique irreducible representation of $\mathfrak{s l}_{3}$ with highest weight $\lambda=(p-q) L_{1}-(q-r) L_{3}=[p-q, q-r]$. We denote this irreducible $\mathfrak{s l}_{3}$-module also by $\mathbb{V}(\lambda)$.

By now, we can answer the following questions: which irreducibles modules $\mathcal{S}_{\lambda_{1}, \lambda_{2}, \lambda_{3}}$ with $\lambda_{1} \geq \lambda_{2} \geq \lambda_{3}$ can be embedded in $\mathcal{M}_{p, q, r}$ and what are their multiplicities? We will show that the following irreducible $\operatorname{Spin}(m)$-modules appear with multiplicity 1 in the decomposition of $\mathcal{M}_{p, q, r}$ :

- $\mathcal{S}_{p+i, q-i, r}$ for every $i=0, \ldots, q-r$, 


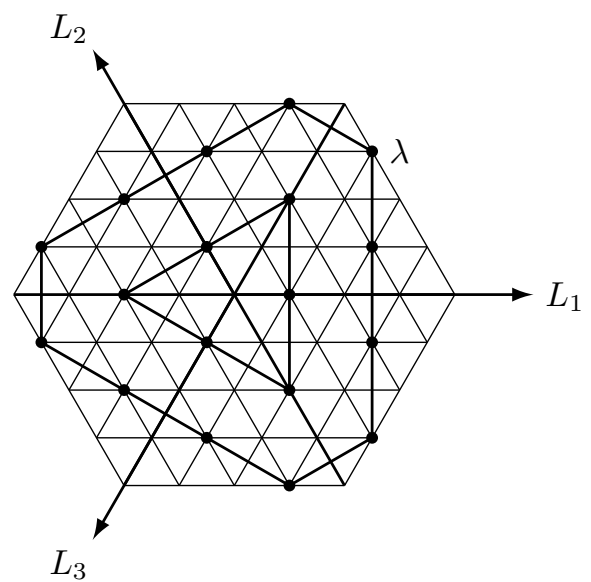

Figure 2: Weights of a representation of $\mathfrak{s l}_{3}$ with highest weight $\lambda$

- $\mathcal{S}_{p, q+i, r-i}$ for every $i=1, \ldots, \min (r, p-q)$,

- $\mathcal{S}_{p+q+r-2 i, i, i}$ for every $i=0, \ldots, r-1$,

- $\mathcal{S}_{p+i, p+i, r+q-p-2 i}$ for every $i=1, \ldots,\lfloor(r+q-p) / 2\rfloor$.

Each of these irreducible $\operatorname{Spin}(m)$-modules corresponds to a unique $\mathfrak{s l}_{3}$-module with respective highest weight

- $[p-q+2 i, q-r-i]$ for every $i=0, \ldots, q-r$,

- $[p-q-i, q-r+2 i]$ for every $i=1, \ldots, \min (r, p-q)$,

- $[p+q+r-3 i, 0]$ for every $i=0, \ldots, r-1$,

- $[0,2 p-q-r+3 i]$ for every $i=1, \ldots,\lfloor(r+q-p) / 2\rfloor$.

If we denote each of these weights by $\lambda$, then the weight $[p-q, q-r]$ corresponding to the highest weight vector $\mathcal{S}_{p, q, r}$ has multiplicity 1 in $\mathbb{V}(\lambda)$. Indeed, if $\lambda=[p-q+2 i, q-r-i]$ or $\lambda=[p-q-i, q-r+2 i]$, the weight $[p-q, q-r]$ lies on the outermost hexagon or triangle and thus has multiplicity 1 . In the other cases $[p-q, q-r]$ lies on inner triangles and has multiplicity 1 since the multiplicities do not change on triangles. In Figure 3 these highest weights are visualized together in the Weyl chamber, denoted by their highest weight as a $\operatorname{Spin}(m)$-module. Note that weights of the form $[p+q+r-3 i, 0]$ and $[0,2 p-q-r+3 i]$ lie on the boundaries of the Weyl chamber.

The fact that these $\operatorname{Spin}(m)$-modules have multiplicity 1 in the decomposition of $\mathcal{M}_{p, q, r}$ is also clear from the Clifford analysis side. As $\left[\left\langle\underline{u}, \underline{\partial}_{x}\right\rangle,\left\langle\underline{v}, \underline{\partial}_{x}\right\rangle\right]=0$ and $\left[\left\langle\underline{v}, \underline{\partial}_{u}\right\rangle,\left\langle\underline{v}, \underline{\partial}_{x}\right\rangle\right]=0$, the only possible embeddings are:

- $\left\langle\underline{u}, \underline{\partial}_{x}\right\rangle^{i} \mathcal{S}_{p+i, q-i, r}$ for every $i=0, \ldots, q-r$,

- $\left\langle\underline{v}, \underline{\partial}_{u}\right\rangle^{i} \mathcal{S}_{p, q+i, r-i}$ for every $i=1, \ldots, \min (r, p-q)$,

- $\left\langle\underline{v}, \underline{\partial}_{x}\right\rangle^{r-i}\left\langle\underline{u}, \underline{\partial}_{x}\right\rangle^{q-i} \mathcal{S}_{p+q+r-2 i, i, i}$ for every $i=0, \ldots, r-1$,

- $\left\langle\underline{v}, \underline{\partial}_{x}\right\rangle^{i}\left\langle\underline{v}, \underline{\partial}_{u}\right\rangle^{i}\left\langle\underline{v}, \underline{\partial}_{u}\right\rangle^{p-q} \mathcal{S}_{p+i, p+i, r+q-p-2 i}$ for every $i=1, \ldots,\lfloor(r+q-p) / 2\rfloor$. 
Note that each space is a subspace of $\mathcal{M}_{p, q, r}$ : they have the correct degree of homogeneity and they are null solutions of the Dirac operators. We will explicitly prove that

$$
\left\langle\underline{v}, \underline{\partial}_{x}\right\rangle^{i}\left\langle\underline{v}, \underline{\partial}_{u}\right\rangle^{p-q+i} \mathcal{S}_{p+i, p+i, r+q-p-2 i} \subset \mathcal{M}_{p, q, r} .
$$

This follows from the definition of simplicial monogenics together with the identities

$$
\begin{aligned}
& {\left[\underline{\partial}_{x},\left\langle\underline{v}, \underline{\partial}_{x}\right\rangle^{i}\left\langle\underline{v}, \underline{\partial}_{u}\right\rangle^{p-q+i}\right]=0} \\
& {\left[\underline{\partial}_{u},\left\langle\underline{v}, \underline{\partial}_{x}\right\rangle^{i}\left\langle\underline{v}, \underline{\partial}_{u}\right\rangle^{p-q+i}\right]=0} \\
& {\left[\underline{\partial}_{v},\left\langle\underline{v}, \underline{\partial}_{x}\right\rangle^{i}\right]=i\left\langle\underline{v}, \underline{\partial}_{x}\right\rangle^{i-1} \underline{\partial}_{x}} \\
& {\left[\underline{\partial}_{v},\left\langle\underline{v}, \underline{\partial}_{u}\right\rangle^{p-q+i}\right]=(p-q+i)\left\langle\underline{v}, \underline{\partial}_{u}\right\rangle^{p-q+i-1} \underline{\partial}_{u} .}
\end{aligned}
$$

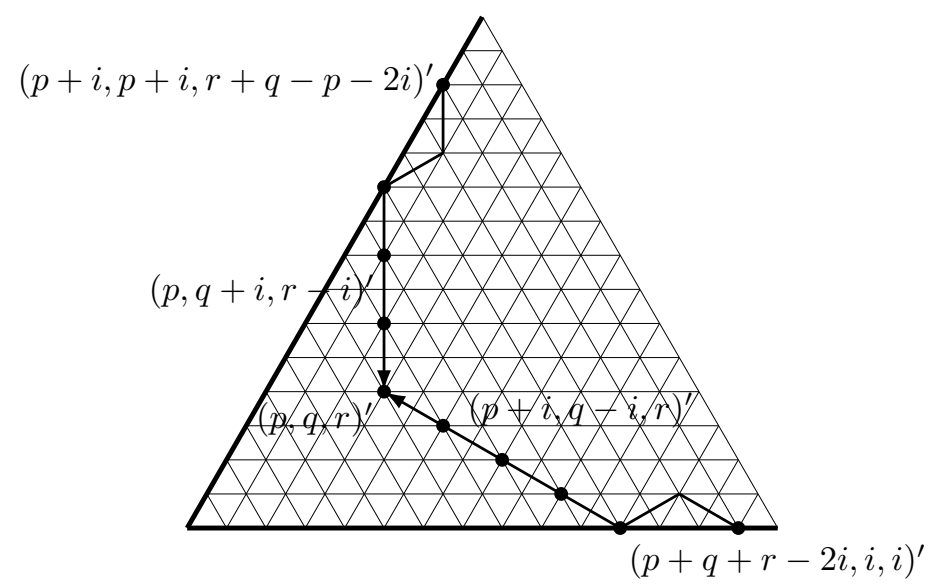

Figure 3: Multiplicity 1

Next we deal with the multiplicities higher than 1 . The following irreducible $\operatorname{Spin}(m)$ modules have multiplicity $(j+1)$ in the decomposition of $\mathcal{M}_{p, q, r}$ :

- $\mathcal{S}_{p+i+j, q-i, r-j}$ for every $i=0, \ldots, q-r$ (if $j \leq r$ ),

- $\mathcal{S}_{p+j, q+i, r-i-j}$ for every $i=1, \ldots, \min (r, p-q)$ (if $\left.j \leq r-i\right)$,

- $\mathcal{S}_{p+q+r-2 i+j, i, i-j}$ for every $i=0, \ldots, r-1$ (if $j \leq i$ ),

- $\mathcal{S}_{p+i+j, p+i, r+q-p-2 i-j}$ for every $i=1, \ldots,\lfloor(r+q-p) / 2\rfloor \quad($ if $j \leq r+q-p-2 i)$.

Each of these irreducible $\operatorname{Spin}(m)$-modules corresponds to a unique $\mathfrak{s l}_{3}$-module with respective highest weight

- $[p-q+2 i+j, q-r-i+j]$ for every $i=0, \ldots, q-r$ (if $j \leq r)$,

- $[p-q-i+j, q-r+2 i+j]$ for every $i=1, \ldots, \min (r, p-q) \quad($ if $j \leq r-i)$,

- $[p+q+r-3 i+j, j]$ for every $i=0, \ldots, r-1 \quad($ if $j \leq i)$,

- $[j, 2 p-q-r+3 i+j]$ for every $i=1, \ldots,\lfloor(r+q-p) / 2\rfloor \quad($ if $j \leq r+q-p-2 i)$. 
If we denote each of these weights by $\lambda$, then the weight $[p-q, q-r]$ corresponding to the highest weight vector $\mathcal{S}_{p, q, r}$, has multiplicity $(j+1)$ in $\mathbb{V}(\lambda)$. This follows from the pattern of multiplicities.

For example, the irreducible $\operatorname{Spin}(m)$-module $\mathcal{S}_{p+2, q, r-2}$ has multiplicity 3 in the decomposition of $\mathcal{M}_{p, q, r}:\left\langle\underline{v}, \underline{\partial}_{x}\right\rangle^{2} \mathcal{S}_{p+2, q, r-2},\left\langle\underline{v}, \underline{\partial}_{x}\right\rangle\left\langle\underline{u}, \underline{\partial}_{x}\right\rangle\left\langle\underline{v}, \underline{\partial}_{u}\right\rangle \mathcal{S}_{p+2, q, r-2}$, and $\left[\left\langle\underline{u}, \underline{\partial}_{x}\right\rangle\left\langle\underline{v}, \underline{\partial}_{u}\right\rangle\right]^{2} \mathcal{S}_{p+2, q, r-2}$ are subspaces of $\mathcal{M}_{p, q, r}$ and the respective embedding maps are linear independent. In general, to find modules of multiplicity $(j+1)$ with $j \geq 1$, we act with the operators $\left\langle\underline{v}, \underline{\partial}_{x}\right\rangle^{k}\left[\left\langle\underline{u}, \underline{\partial}_{x}\right\rangle\left\langle\underline{v}, \underline{\partial}_{u}\right\rangle\right]^{j-k}, 0 \leq k \leq j$, on each space of multiplicity 1 described above. This corresponds with $(j+1)$ different "paths", see Figure 4.



Figure 4: Multiplicities higher than 1

This leads to the decomposition

$$
\begin{aligned}
& \mathcal{M}_{p, q, r}=\bigoplus_{i=0}^{q-r} \bigoplus_{j=0}^{r} \bigoplus_{k=0}^{j}\left\langle\underline{v}, \underline{\partial}_{x}\right\rangle^{k}\left[\left\langle\underline{u}, \underline{\partial}_{x}\right\rangle\left\langle\underline{v}, \underline{\partial}_{u}\right\rangle\right]^{j-k}\left\langle\underline{u}, \underline{\partial}_{x}\right\rangle^{i} \mathcal{S}_{p+i+j, q-i, r-j} \\
& \oplus \bigoplus_{i=0}^{r-1} \bigoplus_{j=0}^{i} \bigoplus_{k=0}^{j}\left\langle\underline{v}, \underline{\partial}_{x}\right\rangle^{k}\left[\left\langle\underline{u}, \underline{\partial}_{x}\right\rangle\left\langle\underline{v}, \underline{\partial}_{u}\right\rangle\right]^{j-k}\left\langle\underline{v}, \underline{\partial}_{x}\right\rangle^{r-i}\left\langle\underline{u}, \underline{\partial}_{x}\right\rangle^{q-i} \mathcal{S}_{p+q+r+j-2 i, i, i-j} \\
& \oplus \bigoplus_{i=1}^{\min (r, p-q)} \bigoplus_{j=0}^{r-i} \bigoplus_{k=0}^{j}\left\langle\underline{v}, \underline{\partial}_{x}\right\rangle^{k}\left[\left\langle\underline{u}, \underline{\partial}_{x}\right\rangle\left\langle\underline{v}, \underline{\partial}_{u}\right\rangle\right]^{j-k}\left\langle\underline{v}, \underline{\partial}_{u}\right\rangle^{i} \mathcal{S}_{p+j, q+i, r-i-j} \\
& \oplus \bigoplus_{i=1}^{L(p+q-r) / 2\rfloor} \bigoplus_{p+q-r-2 i}^{j} \bigoplus_{j=0}^{j}\left\langle\underline{v}_{k=0} \underline{\partial}_{x}\right\rangle^{k}\left[\left\langle\underline{u}, \underline{\partial}_{x}\right\rangle\left\langle\underline{v}, \underline{\partial}_{u}\right\rangle\right]^{j-k}\left\langle\underline{v}, \underline{\partial}_{x}\right\rangle^{i} \\
& \cdot\left\langle\underline{v}, \underline{\partial}_{u}\right\rangle^{p-q+i} \mathcal{S}_{p+i+j, p+i, r+q-p-2 i-j} .
\end{aligned}
$$


In this way the following dimension formula for the triple monogenics is obtained:

$$
\begin{aligned}
\operatorname{dim}_{\mathbb{C}} \mathcal{M}_{p, q, r}= & \sum_{i=0}^{q-r} \sum_{j=0}^{r}(j+1) \operatorname{dim}_{\mathbb{C}} \mathcal{S}_{p+i+j, q-i, r-j} \\
& +\sum_{i=0}^{r-1} \sum_{j=0}^{i}(j+1) \operatorname{dim}_{\mathbb{C}} \mathcal{S}_{p+q+r+j-2 i, i, i-j} \\
& +\sum_{i=1}^{\min (r, p-q)} \sum_{j=0}^{r-i}(j+1) \operatorname{dim}_{\mathbb{C}} \mathcal{S}_{p+j, q+i, r-i-j} \\
& +\sum_{i=1}^{\lfloor(r+q-p) / 2\rfloor} \sum_{j=0}^{r+q-p-2 i}(j+1) \operatorname{dim}_{\mathbb{C}} \mathcal{S}_{p+i+j, p+i, r+q-p-2 i-j}
\end{aligned}
$$

where the Weyl dimension formula (see e.g. [13]) can be used to calculate

$$
\begin{aligned}
\operatorname{dim}_{\mathbb{C}} \mathcal{S}_{p, q, r}= & 2^{n}\left(\begin{array}{c}
p+2 n-3 \\
p+2
\end{array}\right)\left(\begin{array}{c}
q+2 n-4 \\
q+1
\end{array}\right)\left(\begin{array}{c}
r+2 n-5 \\
r
\end{array}\right) \\
& \cdot(2 n+p+q-1)(2 n+p+r-2)(2 n+q+r-3) \\
& \cdot(p-q+1)(p-r+2)(q-r+1) \frac{(2 n-5) !}{(2 n-1) !} \frac{(2 n-5) !}{(2 n-3) !} .
\end{aligned}
$$

Starting from the relation (3) and making use of Grassmann's theorem, we have obtained via quite involved computations another expression for $\operatorname{dim}_{\mathbb{C}} \mathcal{M}_{p, q, r}$ in terms of dimensions of spaces of homogeneous polynomials. By means of Maple, see [19], we were able to verify that both fomulas yield the same result for a number of $(p, q, r)$.

\subsection{Dimension of $\mathcal{M}_{p, q, r}^{s}$}

Recall that in [3] we constructed the unique (up to a multiplicative constant) elliptic, $\operatorname{Spin}(m)$-invariant, first-order differential operator $\mathcal{Q}_{k, l}$, acting between functions taking values in $\mathcal{S}_{k, l}$ :

$$
\mathcal{Q}_{k, l}: \mathcal{C}^{\infty}\left(\mathbb{R}^{m} ; \mathcal{S}_{k, l}\right) \rightarrow \mathcal{C}^{\infty}\left(\mathbb{R}^{m} ; \mathcal{S}_{k, l}\right)
$$

This operator can be seen as a generalization of the Dirac operator $\underline{\partial}_{x}$ and is often referred to as a higher spin Dirac operator. An obvious problem is then to describe the kernel space of this operator. We denote by $\operatorname{Ker}_{h} \mathcal{Q}_{k, l}$ the vector space of the $h$-homogeneous polynomial null solutions of $\mathcal{Q}_{k, l}$. For calculating the dimension of this vector space, we use a generalized version of the classical Cauchy-Kowalewskaia (CK) extension principle in Clifford analysis: considering $\mathbb{R}^{m-1}$ as the hyperplane $x_{m}=0$ in $\mathbb{R}^{m}$, a real-analytic function $f^{*}\left(\underline{x}^{*}\right), \underline{x}^{*}=\sum_{j=1}^{m-1} \underline{e}_{i} x_{i}$, in $\mathbb{R}^{m-1}$ can be uniquely extended to a monogenic function $f\left(\underline{x}^{*}, x_{m}\right)$ in an open neighbourhood in $\mathbb{R}^{m}$ of $\mathbb{R}^{m-1}$, this so-called CK-extension of $f^{*}\left(\underline{x}^{*}\right)$ being given by $f\left(\underline{x}^{*}, x_{m}\right)=\exp \left(-x_{m} \underline{\partial}_{x}^{*}\right) f^{*}\left(\underline{x}^{*}\right)$ with $\underline{\partial}_{x}^{*}=\sum_{i=1}^{m-1} \underline{e}_{i} \partial_{x_{i}}$.

Referring to [4] for details, we mention only the result of the generalized CK extension:

$$
\operatorname{dim}_{\mathbb{C}} \operatorname{Ker}_{h} \mathcal{Q}_{k, l}=\operatorname{dim}_{\mathbb{C}} \mathcal{P}_{h}\left(\mathbb{R}^{m-1}, \mathcal{S}_{k, l}\right)=\operatorname{dim}_{\mathbb{C}} \mathcal{P}_{h}\left(\mathbb{R}^{m-1}\right) \operatorname{dim}_{\mathbb{C}} \mathcal{S}_{k, l}
$$


The dimension of the irreducible $\operatorname{Spin}(m)$-modules $\mathcal{S}_{k, l}$ can be calculated using the Weyl dimension formula (see e.g. [13]) resulting into

$$
\operatorname{dim}_{\mathbb{C}} \mathcal{S}_{k, l}=2^{n}\left(\begin{array}{c}
k+2 n-2 \\
k+1
\end{array}\right)\left(\begin{array}{c}
l+2 n-3 \\
l
\end{array}\right) \frac{(2 n+k+l-1)(k-l+1)}{(2 n-1)(2 n-2)} .
$$

Also in [4] we proved the following decomposition:

Proposition 3 For every $h>k \geq l$, the kernel space $\operatorname{Ker}_{h} \mathcal{Q}_{k, l}$ for the invariant first-order operator $\mathcal{Q}_{k, l}$ decomposes as

$$
\operatorname{Ker}_{h} \mathcal{Q}_{k, l}=\bigoplus_{i=0}^{k-l} \bigoplus_{j=0}^{l} \mathcal{M}_{h-i-j, k-i, l-j}^{s} .
$$

In order to obtain a compact formula for $\operatorname{dim}_{\mathbb{C}} \mathcal{M}_{h, k, l}^{s}$, we follow a pragmatic approach and we apply Proposition 3 on $\operatorname{Ker}_{h} \mathcal{Q}_{k, l}, \operatorname{Ker}_{h-1} \mathcal{Q}_{k-1, l}, \operatorname{Ker}_{h-1} \mathcal{Q}_{k, l-1}$ and $\operatorname{Ker}_{h-2} \mathcal{Q}_{k-1, l-1}$ consecutively:

$$
\begin{aligned}
\operatorname{Ker}_{h} \mathcal{Q}_{k, l}=\mathcal{M}_{h, k, l}^{s} \oplus \bigoplus_{i=1}^{l} \mathcal{M}_{h-i, k, l-i}^{s} \oplus \bigoplus_{i=1}^{k-l} \mathcal{M}_{h-i, k-i, l}^{s} \oplus \bigoplus_{i=1}^{k-l} \bigoplus_{j=1}^{l} \mathcal{M}_{h-i-j, k-i, l-j}^{s}, \\
\operatorname{Ker}_{h-1} \mathcal{Q}_{k-1, l}=\bigoplus_{i=0}^{k-1-l} \bigoplus_{j=0}^{l} \mathcal{M}_{h-1-i-j, k-1-i, l-j}^{s} \\
=\bigoplus_{i=1}^{k-l} \mathcal{M}_{h-i, k-i, l}^{s} \oplus \bigoplus_{i=1}^{k-l} \bigoplus_{j=1}^{l} \mathcal{M}_{h-i-j, k-i, l-j}^{s} \\
=\bigoplus_{i=0}^{k-l+1} \bigoplus_{j=0}^{l-1} \mathcal{M}_{h-1-i-j, k-i, l-1-j}^{s} \\
\operatorname{Ker}_{h-1} \mathcal{Q}_{k, l-1} \\
=\bigoplus_{j=1}^{l} \mathcal{M}_{h-j, k, l-j}^{s} \oplus \bigoplus_{i=1}^{k-l+1} \bigoplus_{j=1}^{l} \mathcal{M}_{h-i-j, k-i, l-j}^{s}
\end{aligned}
$$

and

$$
\operatorname{Ker}_{h-2} \mathcal{Q}_{k-1, l-1}=\bigoplus_{i=1}^{k-l+1} \bigoplus_{j=1}^{l} \mathcal{M}_{h-i-j, k-i, l-j}^{s}
$$

This leads to 
Proposition 4 The dimension of $\mathcal{M}_{h, k, l}^{s}$ is given by

$$
\begin{aligned}
\frac{k(l-1)}{(k-l+1)(2 n-1)(2 n-2)} \operatorname{dim}_{\mathbb{C}} \mathcal{M}_{h, k, l}^{s} \\
=\left(\begin{array}{c}
h+2 n-1 \\
h
\end{array}\right)\left(\begin{array}{c}
k+2 n-2 \\
k-1
\end{array}\right)\left(\begin{array}{c}
l+2 n-3 \\
l-2
\end{array}\right) \frac{(2 n+k+l-1)}{(k+1) l} \\
-\left(\begin{array}{c}
h+2 n-2 \\
h-1
\end{array}\right)\left(\begin{array}{c}
k+2 n-3 \\
k-2
\end{array}\right)\left(\begin{array}{c}
l+2 n-4 \\
l-3
\end{array}\right) \\
\quad \cdot \frac{(2 n+k+l-2)}{(k-1)(k+1)(l-2) l}(2 k l+2 n k+2 n l-l-3 k) \\
+\left(\begin{array}{c}
h+2 n-3 \\
h-2
\end{array}\right)\left(\begin{array}{c}
k+2 n-3 \\
k-2
\end{array}\right)\left(\begin{array}{c}
l+2 n-4 \\
l-3
\end{array}\right) \frac{(2 n+k+l-3)}{(k-1)(l-2)} .
\end{aligned}
$$

Proof. The reasoning above together with the generalized CK-extension (5) leads to

$$
\begin{aligned}
& \operatorname{dim}_{\mathbb{C}} \mathcal{M}_{h, k, l}^{s}=\operatorname{dim}_{\mathbb{C}} \operatorname{Ker}_{h} \mathcal{Q}_{k, l}-\operatorname{dim}_{\mathbb{C}} \operatorname{Ker}_{h-1} \mathcal{Q}_{k-1, l} \\
& -\operatorname{dim}_{\mathbb{C}} \operatorname{Ker}_{h-1} \mathcal{Q}_{k, l-1}+\operatorname{dim}_{\mathbb{C}} \operatorname{Ker}_{h-2} \mathcal{Q}_{k-1, l-1} \\
& =\left(\begin{array}{c}
h+2 n-1 \\
h
\end{array}\right)\left(\begin{array}{c}
k+2 n-2 \\
k-1
\end{array}\right)\left(\begin{array}{c}
l+2 n-3 \\
l-2
\end{array}\right) \\
& \frac{(2 n+k+l-1)(k-l+1)(2 n-1)(2 n-2)}{(k+1) k(l-1) l} \\
& -\left(\begin{array}{c}
h+2 n-2 \\
h-1
\end{array}\right)\left(\begin{array}{c}
k+2 n-3 \\
k-2
\end{array}\right)\left(\begin{array}{c}
l+2 n-3 \\
l-2
\end{array}\right) \\
& \frac{(2 n+k+l-2)(k-l)(2 n-1)(2 n-2)}{(k-1) k(l-1) l} \\
& -\left(\begin{array}{c}
h+2 n-2 \\
h-1
\end{array}\right)\left(\begin{array}{c}
k+2 n-2 \\
k-1
\end{array}\right)\left(\begin{array}{c}
l+2 n-4 \\
l-3
\end{array}\right) \\
& \frac{(2 n+k+l-2)(k-l+2)(2 n-1)(2 n-2)}{(k+1) k(l-2)(l-1)} \\
& +\left(\begin{array}{c}
h+2 n-3 \\
h-2
\end{array}\right)\left(\begin{array}{c}
k+2 n-3 \\
k-2
\end{array}\right)\left(\begin{array}{c}
l+2 n-4 \\
l-3
\end{array}\right) \\
& \cdot \frac{(2 n+k+l-3)(k-l+1)(2 n-1)(2 n-2)}{(k-1) k(l-2)(l-1)} \text {. }
\end{aligned}
$$

This is then simplified by combining the second and third term, leading to the desired result. Note that we also have

$$
\operatorname{dim}_{\mathbb{C}} \mathcal{M}_{h, k, l}^{s}=\sum_{i=0}^{k-l} \sum_{j=0}^{l} \operatorname{dim}_{\mathbb{C}} \mathcal{S}_{h+i+j, k-i, l-j} .
$$

We used Maple (see [19]) to verify that these two expressions for $\operatorname{dim}_{\mathbb{C}} \mathcal{M}_{h, k, l}^{s}$ are indeed equal.

\section{References}

[1] Brackx, F., Bureš J., De Schepper H., Eelbode D., Sommen F. and Souček V., Fundaments of Hermitean Clifford Analysis. Part I: Complex structure, Compl. Anal. Oper. Theory 1(3), 2007, 341-36 
[2] Brackx, F., Delanghe, R., Sommen, F., Clifford Analysis, Research Notes in Mathematics 76, Pitman, London, 1982.

[3] Brackx, F., Eelbode, D., Van de Voorde, L., Invariant operators between spaces of simplicial monogenics, submitted.

[4] Brackx, F., Eelbode, D., Van de Voorde, L., The polynomial null solutions of a higher spin Dirac operator in two vector variables, submitted.

[5] Branson, T., Stein-Weiss operators and ellipticity, J. Funct. Anal. 151 No. 2 (1997), pp. 334-383.

[6] Bureš, J., Sommen, F., Souček, V., Van Lancker, P., Rarita-Schwinger type operators in Clifford analysis, Journal of Funct. Anal. 185, pp. 425-456.

[7] Bureš, J., Sommen, F., Souček, V., Van Lancker, P., Symmetric analogues of RaritaSchwinger equations, Ann. Glob. Anal. Geom. 21 No. 3 (2001), pp. 215-240.

[8] Constales, D., Sommen. F., Van Lancker, P., Models for irreducible representations of Spin $(m)$, Adv. Appl. Clifford Algebras 11 No. S1 (2001), pp. 271-289.

[9] Delanghe, R., Sommen, F., Souček, V., Clifford analysis and spinor valued functions, Kluwer Academic Publishers, Dordrecht, 1992.

[10] Delanghe, R., Clifford analysis: history and perspective, Computational Methods and Function Theory 1 (2001), pp. 107-153.

[11] Eelbode, D., Van de Voorde, L., A toy model for higher spin Dirac operators, Physics of Atomic Nuclei, 2010, Vol. 73, No. 2, pp. 282-287.

[12] Fegan, H. D., Conformally invariant first order differential operators, Quart. J. Math. 27 (1976), pp. 513-538.

[13] Fulton, W., Harris, J., Representation theory : a first course, Springer-Verlag, New York, 1991.

[14] Gilbert, J., Murray, M.A.M., Clifford algebras and Dirac operators in harmonic analysis, Cambridge University Press, Cambridge, 1991.

[15] Guerlebeck, K. and Sproessig, W., Quaternionic and Clifford Calculus for Physicists and Engineers, Wiley, Chichester, 1998.

[16] Hall, Brian C., Lie Groups, Lie Algebras, and Representations : An Elementary Introduction, Springer, New York, 2003.

[17] Humphreys, J., Introduction to Lie algebra and representation theory, Springer-Verlag, New York, 1972.

[18] Lavicka, R., Souček, V, Massless field operators in Clifford analysis.

[19] Maple (tool for mathematics and modeling), Waterloo Maple Inc., http://www.maplesoft.com/products/Maple

[20] Molev, A.I., Weight bases of Gelfand-Tsetlin type for representations of classical Lie algebras, Journ. Phys. A 33, No. 22 (2000), pp. 4143-4158. 
[21] Raeymaekers, T., $\mathfrak{s l}_{3}$ and its representations: applications in Clifford analysis (in Dutch), Master thesis, Ghent University, 2009.

[22] Stein, E.W., Weiss, G., Generalization of the Cauchy-Riemann equations and representations of the rotation group, Amer. J. Math. 90 (1968), pp. 163-196. 